Voix et Images

voixetimages

\title{
Quarante ans d'études québécoises en Grande-Bretagne
}

\section{Rachel Killick}

Volume 27, numéro 1 (79), automne 2001

Fernand Dumont

URI : https://id.erudit.org/iderudit/201594ar

DOI : https://doi.org/10.7202/201594ar

Aller au sommaire du numéro

\section{Éditeur(s)}

Université du Québec à Montréal

\section{ISSN}

0318-9201 (imprimé)

1705-933X (numérique)

Découvrir la revue

\section{Citer cet article}

Killick, R. (2001). Quarante ans d'études québécoises en Grande-Bretagne. Voix et Images, 27(1), 158-164. https://doi.org/10.7202/201594ar d'utilisation que vous pouvez consulter en ligne.

https://apropos.erudit.org/fr/usagers/politique-dutilisation/ 


\title{
Quarante ans d'études québécoises en Grande-Bretagne
}

\author{
Rachel Killick, Université de Leeds
}

C'est à partir du séjour de Cedric May à McDonald College de l'Université McGill (1961-1963) que l'on peut retracer le développement en Grande-Bretagne des études québécoises. À l'époque, les programmes universitaires de français en GrandeBretagne ne tenaient compte que de la littérature de la Métropole; le jeune professeur britannique se trouvait au Québec au début de la Révolution tranquille et il a assisté à la grande éclosion littéraire et culturelle de l'époque. De retour en GrandeBretagne et alors maitre de conférences au Département d'études françaises de l'Université de Birmingham, May a mené de front ses propres recherches québécoises et l'implantation institutionnelle en GrandeBretagne des recherches québécoises et canadiennes, implantation qui s'est faite par l'établissement d'un volet canadien à l'intérieur du programme d'études américaines. Avec la collaboration d'autres collègues universi- taires, ses efforts ont abouti, en 1981, à la création d'un Centre régional d'études canadiennes ${ }^{1}$. May a publié la même année Breaking the Silence: The Literature of Quebec ${ }^{2}$, texte fondateur des études québécoises en Grande-Bretagne, qui avait pour objectif de sensibiliser les professeurs de lycée et d'université à l'intérêt culturel et esthétique de la littérature québécoise, et d'encourager l'inclusion de textes québécois dans les programmes d'enseignement. L'ouvrage effectue un tour d'horizon de la littérature québécoise, la situant dans son contexte historique et culturel et faisant ressortir ses préoccupations majeures par l'analyse serrée d'un choix d'œuvres et d'auteurs constituant un éventail géographique, social, culturel et formel aussi large que possible. Il s'agit de textes d'une douzaine d'auteurs: Émile Nelligan, Alain Grandbois, Germaine Guèvremont, Paul-Émile Borduas, André Langevin, Yves Thériault, Gérard 
Bessette, Jacques Godbout, Jacques Poulin, Jacques Brault, Jean Barbeau et Roch Carrier. Trois ans plus tard, toujours dans le but de révéler les richesses plus ou moins inconnues en Grande-Bretagne de la littérature québécoise, May revient à Godbout avec $A$ Student's and Teacher's Guide to Jacques Godbout's "Salut Galarneau " 3 . D'autres publications suivront à propos de la poésie et du roman québécois ainsi que sur la littérature franco-canadienne du Manitoba et de l'Acadie ${ }^{4}$. Bien qu'il soit retraité depuis 1988, May s'intéresse toujours au Québec, comme l'indique notamment sa participation au colloque Regenerations (Université de Leeds, 1999) où il a présenté une communication intitulée "Gaston Miron: Artisan of the Emerging Voice $^{5}$ ".

Si les études québécoises en Grande-Bretagne ont été introduites dans le cadre institutionnel des études américaines, elles se sont progressivement insérées dans des programmes élargis d'études françaises. Le rôle joué par l'Écosse est particulièrement intéressant: la perception d'une similitude culturelle entre l'Écosse et le Québec - deux sociétés minoritaires face à une société anglaise ou anglophone dominante - favorise une sympathie croissante pour les productions de la culture québécoise. Le programme d'études françaises de l'Université d'Édimbourg comprenait déjà, en 1977, un demi-module en littérature francocanadienne et, dans les années 1980, Ian Lockerbie (Université de Stirling) a pu rejoindre un public plus large. Il organisa, en 1989, un colloque faisant état du théâtre de Michel Tremblay au moment où, dans le cadre de la saison New Scottish Theatre, Tom McGrath présentait une lecture dramatique des Belles-Soeurs dans la traduction, depuis lors célèbre, de Martin Bowman et de Bill Findlay ${ }^{6}$.

Avec les troubles en Algérie et la diaspora des universitaires maghrebins, dans les années 1990, s'accroît l'intérêt pour les études francophones hors de l'Hexagone. Pour le développement des études québécoises en Grande-Bretagne, il s'agit cependant d'un progrès relatif, malgré les liens déjà signalés par Cedric May, en 1981, entre la pensée du groupe Parti pris, les théories de la décolonisation de Sartre, de Fanon, de Memmi et de Berque et la vision interculturelle plus positive de Césaire et de Senghor $^{7}$. Les discours anglophones de l'Amérique du Nord, qui ont tendance à occulter le discours québécois, se doublent maintenant des discours des pays d'Afrique ou des Caraibes qui, eux aussi, compte tenu de leur évolution propre et tout francophones qu'ils soient, risquent de lui faire écran. Aux centres d'études canadiennes s'ajoutent maintenant les centres d'études francophones, celui de l'Université de Leeds, par exemple, fondé en 1997. La Grande-Bretagne a tout de même la chance d'être dotée d'un Centre d'études québécoises, situé à l'Université de Leicester. Établi en 1998, avec l'appui du Québec, il est dirigé par Christopher Rolfe et bénéficie aussi des services d'un adjoint, poste occupé pendant les deux premières années par Céline Gagnon, doctorante québécoise, spécialiste de l'œuvre d'Yves Thériault.

Chaque année ont lieu en Grande-Bretagne deux colloques qui intéressent particulièrement les québécistes: la grande réunion de 
trois jours de la British Association for Canadian Studies (BACS), fondée par Cedric May et d'autres canadianistes en 1975; et la Journée d'études du Groupe de recherches et d'études sur le Canada français (GRECF), tenue au sein de la BACS à l'initiative de Cedric May pour donner plus de cohésion aux activités des québécistes dans tous les domaines. La réunion de la BACS inclut toujours, en plus des communications individuelles des canadianistes et québécistes, une conférence plénière prononcée par une sommité québécoise. L'invité d'honneur, en 2000, fut Claude Ryan; en 2001, Lise Bissonnette. La journée du GRECF a accueilli, en 2000, Pierre Nepveu; en 2001, le politologue Michel Seymour. Par ailleurs, une journée d'études a été organisée tout récemment au Centre d'études québécoises de l'Université de Leicester par Céline Gagnon avec l'écrivaine Ying Chen, comme invitée d'honneur, et des communications sur le théâtre et le cinéma québécois ont été prononcées par Michael Cardy (Université de Wales, Swansea), Marie-Christine Lesage (Université de Montréal), Fergus Morrin (Université de Nottingham) et Craig Moyes (Kings' College, London). À Leeds, dans le cadre du Centre d'études francophones, nous organisons tous les deux ans un colloque international. En 1999, Jacques Allard a prononcé la conférence plénière sur le roman québécois. Nous avons pu accueillir aussi Micheline Cambron et Marie-Christine Lesage de l'Université de Montréal.

En septembre 2001, a eu lieu notre $3^{\mathrm{e}}$ colloque international "Racines et branches: sociétés et cultures francophones de l'Europe et des Améri- ques", qui a accueilli les conférenciers québécois Noël Audet, Jacques Allard, Daniel Chartier et Lise Gauvin et le linguiste belge, Raymond Renard. Autre phénomène récent et prometteur: la présence d'intervenants québécois, en 2000, au colloque de la BACS à Édimbourg et au colloque annuel de la Society for French Studies (l'Association nationale des études françaises en Grande-Bretagne) à Londres. Avec le soutien de la Délégation générale du Québec à Londres, nous profitons aussi, dans nos différentes universités, des visites des Québécois et québécistes en tournée britannique et européenne. À titre d'exemple, nous avons reçu les universitaires Jocelyn Létourneau et Kenneth McRoberts ainsi que l'ecrivain Marco Micone, pour qui nous avons mis en scène, à Leeds, une lecture dramatique de sa pièce Gens du silence.

C'est surtout par les arts du spectacle que le Québec littéraire et culturel s'est fait connaitre en GrandeBretagne. Échappant à toute contrainte linguistique, le Cirque du Soleil jouit d'une grande réputation tandis que la troupe La La La Human Steps est connue de tous les amateurs de danse. Les amateurs et les spécialistes de théâtre et de cinéma suivent avec beaucoup d'intérêt le travail de Robert Lepage. Les pièces de Michel Tremblay ont aussi du succès, et on a parfois la possibilité de voir des pièces d'autres dramaturges tels que Marie Laberge ou Daniel Danis ${ }^{8}$. Il existe un lien particulier entre Tremblay "the greatest Scottish playwright Scotland never had "et le public écossais, mais ses pièces attirent aussi le public anglais pour leur mélange caractéristique de réalisme, de 
surréalisme, de fantaisie et de poésie. En 2000, à Leeds, des étudiants de premier cycle en études théâtrales ont produit une interprétation magnifique des Belles-Soeurs dans la traduction écossaise de Martin Bowman et Bill Findlay.

Les chercheurs en GrandeBretagne disposent en principe non seulement des textes originaux mais aussi des traductions des auteurs québécois les plus en vue, ce qui devrait faciliter l'accès à la littérature québécoise à tous ceux qui s'intéressent à la production contemporaine. Mais, en fait, à part un ou deux auteur(e)s de renommée internationale (Nicole Brossard, par exemple), cette littérature reste mal connue hors quelques départements d'études françaises. Il faudrait souligner cependant le rôle exceptionnel des traducteurs Bowman et Findlay. À l'encontre d'autres traductions de textes québécois, peu disponibles dans les bibliothèques britanniques, encore moins dans les librairies, les leurs, exposées directement aux spectateurs, assurent un échange culturel réussi entre le Québec et la Grande-Bretagne. D'ailleurs, ils ne limitent pas leurs activités aux seules pièces de Tremblay. Ils ont également réalisé des traductions de Jeanne-Mance Delisle (Un "reel" ben beau, ben triste/The Reel of the Hanged Man) et de René-Daniel Dubois (Les Feluettes/The Skelfs). Inversant le processus de traduction, ils ont aussi assuré la transmission au Québec du film britannique Trainspotting, transformant en joual l'écossais original. Au-delà de la scène, leurs traductions donnent lieu à une réflexion stimulante sur les parallèles entre les deux cultures et sur leurs spécificités, à la fois de la part des traducteurs eux-mêmes sous forme d'articles et de présentations dans des colloques scientifiques sur la traduction (par exemple, celui du groupe TRIO de l'Université d'Oxford) et de la part de commentateurs universitaires, tel David Kinloch (Université de Strathclyde) ${ }^{9}$.

Dans le domaine des recherches littéraires, les contributions britanniques commencent à se multiplier, consolidant le travail pionnier de May. Il faudrait surtout faire état de la contribution soutenue de Christopher Rolfe, directeur du GRECF pendant plus de dix ans, animateur de nombreuses journées d'études à l'Institut français et à l'Institut des langues romanes, rédacteur d'une bibliographie critique annuelle des études littéraires sur le Canada français et auteur de plusieurs études sur des sujets aussi variés que les romanciers québécois des années 1950, le polar québécois et la production d'estampes et de gravures au Québec, domaine où il est lui-même actif ${ }^{10}$. L'année 2000 a vu la parution de deux monographies, Quebec National Cinema de Bill Marshall (Université de Glasgow) et Siting the Quebec Novel de Rosemary Chapman (Université de Nottingham) ${ }^{11}$, tandis que la publication accélérée de guides de lecture témoigne de la croissance du public étudiant: Peter Noble (Université de Reading), Anne Hébert: Les Fous de Bassan (Glasgow French Texts, 1995); Guy Snaith (Université de Liverpool), Roch Carrier: La Guerre. Yes sir! (London, Bristol Classical Press, 2000); Michael Cardy (Université de Wales, Swansea), Michel Tremblay: Les Belles-Soeurs and À toi pour toujours ta Marie-Lou (Londres, Grant \& Cutler, sous 
presse). Dans la même perspective, mon édition des Belles-Sœurs (London, Bristol Classical Press, 2000) propose aux professeurs et étudiants des départements britanniques d'études françaises un texte français et un appareil critique en anglais comprenant une analyse détaillée de la pièce et la situant dans son contexte québécois. On remarque aussi la présence du Québec non seulement dans des périodiques ${ }^{12}$ mais également dans des livres issus de différents domaines de recherche. J'ai déjà cité l'ouvrage de Kinloch et Price. La monographie de Christopher Robinson, Scandal in the Ink: Male and Female Homosexuality in Twentietb-Century Literature (London, New York, Cassell, 1995) en est un autre exemple, avec un chapitre consacré à Michel Tremblay.

Une connaissance de l'évolution du travail critique dans les études québécoises tant britanniques qu'internationales est assurée par la bibliographie critique annuelle, le Year's Work in Modern Language Studies, publiée par la Modern Humanities Research Association. Avant 1988, les quelques références aux livres et aux articles ayant trait aux auteurs québécois étaient tout simplement incorporées sous la rubrique "Littérature du $20^{\mathrm{e}}$ siècle (post1945)». Depuis, progrès significatif, le Canada français a droit à une section séparée $^{13}$. Il allait de soi que ce premier tour d'horizon revînt à "Mr. Quebec Literature "Cedric May ${ }^{14}$. Dès l'année suivante, Christopher Rolfe a pris la relève, tâche qu'il continue d'exercer avec beaucoup de dévouement. Les québécistes en GrandeBretagne disposent aussi de l'œuvre bibliographique magistrale French
Quebec: Imprints in French from Quebec 1764-1990 in the British Library (D.J. McTernan [ed.], 2 volumes, London, British Library, 1992). Quant aux périodiques, le Bulletin of Canadian Studies, fondé par la BACS en 1977, et son successeur, le British Journal of Canadian Studies, paraissent deux fois l'an et publient des articles sur la société et la culture canadiennes et québécoises. Les articles sur le Québec se préoccupent le plus souvent de littérature, mais certains ont trait à la culture entendue de façon plus large (presse populaire, cinéma, etc.) et aux questions politiques et sociales. À Leeds, l'International Journal of Francopbone Studies, fondé en 1998 et dirigé par Kamal Salhi, du Centre d'études francophones, comprend outre des articles sur des pays d'Afrique et des Caraïbes, des articles ayant trait à la littérature québécoise. Une nouvelle publication, Focus. Essays on Quebec Society and Culture, soutenue par la BACS et dirigée par Christopher Rolfe et la politologue Ines Molinaro (Université de Cambridge), regroupe les communications présentées à la journée d'études annuelle du GRECF. En plus des périodiques et des publications spécialisés, le Québec commence à faire son apparition dans des périodiques généralistes de langues et de littératures tel le très respecté Modern Language Review, avec des comptes rendus et parfois un article. On a vu aussi le Times Literary Supplement, hebdomadaire culturel national, publier quelques comptes rendus de textes québécois et leur assurer ainsi un rayonnement plus large.

Ces efforts de recherches et de vulgarisation sont reliés à des cours de littérature et de société et culture 
québécoises à la licence. Des programmes d'études françaises comportant un ou deux modules sur le Québec existent à Leeds, Leicester, Liverpool, Nottingham, Reading et Sheffield, et, en Écosse, à Glasgow et à Édimbourg. Dans certains cas, les étudiants qui doivent tous effectuer un séjour dans un pays francophone peuvent être accueillis dans une université québécoise. Sheffield, par exemple, envoie des étudiants à l'Université Laval et à l'Université de Sherbrooke; Leeds a des programmes d'échange avec l'Université du Québec à Trois-Rivières et avec l'Université de Montréal et Glasgow envoie des étudiants à l'Université du Québec à Montréal et à l'Université McGill. Parfois, aussi, des étudiants parviennent à décrocher un poste d'assistant d'anglais, ce qui leur donne la possibilité de vivre un an au sein de la société québécoise, mais le nombre de ces postes est malheureusement limité. En Grande-Bretagne, les programmes de maîtrise sont beaucoup moins développés qu'en Amérique du Nord. Il existe pourtant des possibilités de poursuivre des études québécoises au cycle supérieur, telle la maîtrise en études francophones de l'Université de Leeds, qui comporte une option québécoise, et la maîtrise en théorie critique de l'Université de Nottingham, qui peut faire appel à la littérature québécoise. Les bourses étant très peu nombreuses, le doctorat en Grande-Bretagne n'attire en général qu'une poignée d'étudiants dans le domaine des arts et des lettres, qui visent presque tous une carrière universitaire.

Dans ces conditions, le choix d'un sujet québécois n'est pas sans conséquence pour l'étudiant britanni- que. Le sujet retenu doit s'accorder avec un profil de recherches souple, susceptible d'être intégré dans les divers programmes des départements d'études françaises où le doctorant finira par poser sa candidature. Les thèses traitant de l'écriture féminine, de l'inscription de l'histoire dans les œuvres d'imagination, du cinéma, de la littérature amérindienne francophone et de la littérature migrante ont actuellement la préférence des étudiants.

Les spécialistes de la littérature québécoise ont pour compagnons de route des québécistes et des canadianistes dans d'autres domaines ${ }^{15}$. Les études québécoises proposent à un public britannique un domaine riche où s'entrecroisent les grandes questions linguistiques, culturelles, politiques et sociales du monde moderne. C'est ce qui explique, chez un noyau de chercheurs convaincus et un nombre grandissant d'étudiants, un enthousiasme pour le Québec, société francophone et multiculturelle de l'Amérique du Nord.

1. Il existe cinq Centres régionaux d'études canadiennes au Royaume-Uni, soit à l'Université de Birmingham, à la Queen's University of Belfast, à l'Université d'Édimbourg, à I'Université de Leeds et à Birkbeck College (Université de Londres).

2. Université de Birmingham: Centre régional d'études canadiennes, 1981.

3. Université de Birmingham: Centre régional d'études canadiennes, 1984.

4. Voir, dans le Bulletin of Canadian Studies, vol. 1-9 (1977-1985), une série d'articles comprenant des tours d'horizon de la tittérature et la culture franco-canadiennes, ainsi que des études ponctuelles sur la poésie québécoise (Saint-Denys Garneau, Gaston Miron, Fernand Ouellette), sur Gabrielle Roy, Antonine Maillet, Jacques Poulin et sur l'historien Michel Brunet.

5. Les actes du colloque seront publiés dans un numéro spécial du British Journal of Canadian Studies, édité par Susan Billingham 
(Université de Nottingham), spécialiste de la littérature canadienne anglophone.

6. C'est Lockerbie qui avait fait connaître la pièce de Tremblay à Tom McGrath.

7. Cedric May, Breaking the Silence, p. 31.

8. Voir mon article, - La réception du théâtre québécois en Grande-Bretagne: une perspective anglaise ', L'Annuaire théâtral, $\mathrm{n}^{\circ} 27$, printemps 2000, p. 204-220.

9. "Songs of the Flute-Bone: Scots Translations of Michel Tremblay , David Kinloch et Richard Price (dir.), Une nouvelle alliance: France et la littérature écossaise moderne, Grenoble, ELLUG, 1999.

10. Voir, à titre d'exemple, *Des livres importants à redécouvrir", (sur Roger Viau et Jean-Jules Richard), British Journal of Canadian Studies, vol. II, $\mathrm{n}^{\circ} 2$, p. 267-276; * Detecting Change: Women Crime Writers in Quebec n, (sur Monique Lepage et Chrystine Brouillet), British Journal of Canadian Studies, vol. X, $\mathrm{n}^{\circ} 1,1995$, p. 127-138; "Of Brawls and Riots: (Pre)-Figuring Social Change in the Quebec Novel of the 50s *, Journal of the Institute of Romance Studies, 3, 1995, p. 305-316; *A Collaborative (Ad) venture: Metropolitan Museum *, (au sujet de la collaboration du poète Robert Choquette et du graveur Edwin Holgate) British Journal of Canadian Studies, vol. XIII, no 1, 1998, p. 1-16.

11. Rosemary Chapman, Siting the Quebec Novel, Montréal/Kingston, McGill/Queen's University Press, 2001 et Peter Lang, 2000. Chap- man vient de signer aussi la partie intitulée - Amérique du Nord. dans Francophone Literatures. A Literary and Linguistic Companion, Malcolm Offord et al., London/New York, Routledge, 2001, p. 211-277.

12. À noter, parmi les articles, ceux de nouvelles recrues, telles Ceri Morgan (.It's the End of the World as We Know It: Quebec's Remembered Landscapes ", British Journal of Quebec Studies, vol. XIV (i), 1999, p. 52-62) et Catherine Scott ("Swollen with lightning like revolt, bursting with anger $[\ldots]$ they dance wild dances": Theatre as Transgressive Space in Quebec's Women's Writing in the 1970s *, New Readings, 1999, p. 61-70).

13. L'Afrique et le Maghreb ont eu droit de même, en 1988, à une section à part. En 1993, une section distincte sur la littérature des Caraïbes a été inaugurée.

14, Voir la biographie professionnelle de Cedric May dans le British Journal of Canadian Studies, vol. III, n ${ }^{\circ} 2,1988$, p. 205.

15. Signalons, entre autres, les recherches historiques de Colin Coates et les recherches politiques de James Kennedy (tous les deux de l'Université d'Ếdimbourg) ou les recherches sur les Premières Nations (par exemple, Aboriginal People and Other Canadians: Shaping New Relationships, projet de groupe dirigé par Roy Todd dans le cadre du Centre d'études régional de Leeds, actuellement sous presse à l'Université d'Ottawa). 